\title{
Classification and regression tree analysis in acute coronary syndrome patients
}

\author{
Heng-Hsin Tung ${ }^{1,2^{*}}$, Chiang-Yi Chen ${ }^{3}$, Kuan-Chia Lin ${ }^{1}$, Nai-Kuan Chou ${ }^{3}$, Jyun-Yi Lee ${ }^{4}$, \\ Daniel L. Clinciu, ${ }^{5,6}$, Ru-Yu Lien ${ }^{1,7}$
}

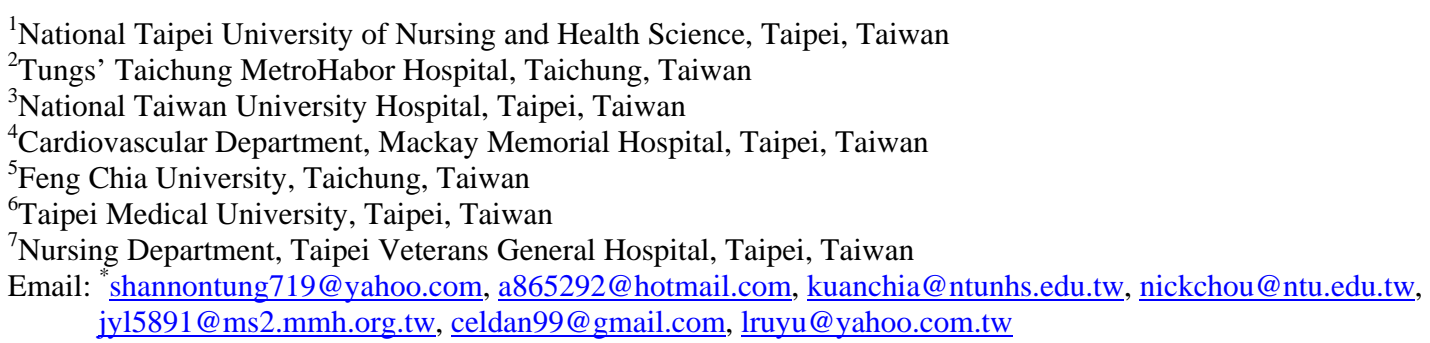

Received 9 April 2012; revised 8 May 2012; accepted 20 May 2012

\begin{abstract}
Objectives: The objectives of this study are to use CART (Classification and regression tree) and stepwise regression to 1 ) define the predictors of quality of life in ACS (acute coronary syndrome) patients, using demographics, ACS symptoms, and anxiety as independent variables; and 2) discuss and compare the results of these two statistical approaches. Background: In outcome studies of ACS, CART is a good alternative approach to linear regression; however, CART is rarely used. Methods: A descriptive survey design was used with 100 samples recruited. Result and Conclusions: Anxiety is the most significant predictor and also a stronger predictor than symptoms of ACS for the quality of life. The anxiety level patients experienced at the time heart attack occurred can be used to predict quality of life a month later. Furthermore, the majority of ACS patients experienced a moderate to high level of anxiety during a heart attack.
\end{abstract}

Keywords: CART; Stepwise Regression; Acute Coronary Syndrome; Anxiety; Quality of Life

\section{INTRODUCTION}

Acute coronary syndrome (ACS) includes any group of syndromes such as unstable angina (UA), non-ST-elevation MI (NSTEMI) and ST-elevation MI (STEMI) [1]. In United States the direct and indirect costs of coronary heart disease, which includes ACS, were about \$165.4 billion in 2009 [1]. In Taiwan, heart disease is among the

\footnotetext{
${ }^{*}$ Corresponding author.
}

top two leading causes of death, and in 2011, one person died of heart disease every 33 minutes and 32 seconds [2].

Patients with ACS experience chest pain, dyspnea, nausea, vomiting, diaphoresis, fatigue, dizziness, and the pain can radiate to the neck, chin, and left shoulder [3]. Dec Von (2008) [4] illustrated that in regard to gender, men commonly experience more chest pain while women are more likely to report indigestion, palpitations, nausea, weakness, and numbness of the extremities. Shih et al. (2009) [5] indicated women experience more back pain, palpitations, nausea/vomiting, and loss of appetite, compare to men. To sum up, women tend to experience more associated symptoms than men. Additionally, a greater number of ACS symptoms and more chest pain are associated with a higher level of anxiety $[5,6]$.

Anxiety can be the consequence of a coronary disease as well as playing a contributing role [7-9]. Koivula et al. (2001) [10] reported that nearly half of their patients had medium to high anxiety while awaiting cardiac surgery. Other studies have shown that anxiety levels decrease after a cardiac event, such as a heart attack, angina, or surgery [11,12], while Andrew (2000) [13] found that $40 \%-50 \%$ of patients still experienced anxiety following heart surgery, and $20 \%$ of cardiac patients remain anxious even one year after surgery [14]. Additionally, after cardiac surgery women tend to suffer higher levels of anxiety than men do [15].

Both ACS symptoms and anxiety play important roles in the quality of life. The more ACS symptoms are experienced by patients, the lower the quality of life [1618]. Anxiety levels increase with a cardiac event, and higher anxiety levels are associated with a lower quality of life and poor outcomes [19-25]. Therefore, patients 
who have fewer ACS symptoms and lower anxiety are more likely to achieve a better quality of life.

Classification and regression tree (CART) analysis has gradually become a popular statistical technique in health sciences because the decision tree is easy to understand and provides a clear means to make clinical decisions [26]. Unlike traditional regression analysis, CART is able to manage data that is not normally distributed, as well as handling different types of independent variables, including continuous, categorical, or a mix of both. CART also can manage complicated interactions between variables because non-predictable independent variables are not included in the final tree neither influences the results. In comparison, linear regression can provide a single, best-fit line through parametric data without bias. While linear regression is an acceptable and commonly used analytic tool, CART is considered a good alternative to analyze data [26,27].

In short, symptoms and anxiety are important correlates of quality of life in ACS patients. CART is an alternative approach for data analysis to linear regression in determining the predictors of the dependent variable. In outcome studies of ACS, however, CART is rarely used. Thus, the objectives of this study are to 1) use CART and stepwise regression in defining the predictors of quality of life in ACS patients, using demographics, ACS symptoms, and anxiety as independent variables; and 2) to discuss and compare the results of these two statistical approaches.

\section{METHODS}

A descriptive survey design was used in this study. Participants were recruited between August 2010 and February 2011, using the following criteria: 1 ) hospitalized in a cardiac unit ward, 2) between 20 and 80 years old, 3) diagnosed as having ACS, 4) experienced a cardiac attack within a month, and 5) able to communicate. Those who had cardiac surgery within three months or who had psychiatric problems were excluded. G*Power, Version 3.1 (Department of Criminology, University of Melbourne, Parkville, Victoria 3010, Australia), was utilized to estimate sample size. The analysis determined that at least 82 participants should be sampled, based on the significance level being set at $\alpha=0.05$, the statistical power at $(1-\beta)=0.80$, and effect size at 0.30 .

\subsection{Instruments}

Demographic questionnaire. This questionnaire included items related to age, gender, education, employment status, living status (with whom the patient was living), primary caregiver, religion, tobacco use, alcohol use and exercise habit. There are six different frequency of exercise habit (daily, 3 - 6 times a week, 1 - 2 times a week, 3
- 4 per month, 1 - 2 times per month and no regular exercise) with more than 30 mins duration per time. Selfperceived family support and self-perceived environmental support, which were measured on a 10-point Likert scale, with a higher score indicating higher satisfaction.

The Charlson Comobidity Index (CCI) was used to assess a co-existing disease. CCI is a measure of the probability of one-year mortality based on comorbidity in patients hospitalized in general hospitals. CCI consists of a list of 19 comorbid conditions weighted according to the risk of death, with the scores summed to produce an index score. The potential scores of this index range from 0 to 37, with higher scores indicating greater severity of the patient's health condition [28-31].

Symptoms of Acute Coronary Syndrome Inventory (SACSI). This inventory is used to assess ACS symptoms and includes three sections. In the first section, 20 symptoms are described, and patients, using a 5-point Likert scale, rate the symptom, with 0 indicating no symptoms, 1 indicating mild, 2 indicating moderate, 3 indicating severe, and 4 indicating very severe. In the second section, 14 locations in the body, where pain or discomfort may be experienced, are presented, and patients indicate "yes" or "no". In the third section, 14 different types of pain severity are presented, and patients indicate "yes" or "no" [4].

The original SACSI was translated into Chinese by a nurse with 10 years of experience whose primary language is Chinese. Next, a bilingual individual with doctoral preparation did the back translation into English to compare it with the original questionnaire. The Chinese version also was reviewed and revised by an expert panel that included two master's-prepared nurses, one nursing faculty member with a doctorate, and one cardiologist. In this study, the content validity index (CVI) of the final Chinese version of the SACSI was 0.97, and Cronbach's alpha was 0.56 .

State Trait Anxiety Inventory (STAI). The Chinese version of the STAI was used to measure anxiety. The scale consists of 20 state anxiety items and 20 trait anxiety items. State anxiety is a temporary feeling of anxiety, while trait anxiety is a tendency to be anxious. Respondents use a 4-point Likert scale ( 0 = none, $1=$ minor, 2 = moderate, and $3=$ severe) to indicate the degree of anxiety. The total score ranges from 20 to 80 , with a higher score indicating a higher level of anxiety. The Chinese version of the STAI has been reported to have good psychometric properties, and the STAI has been used with the cardiac population [32]. In this study, Cronbach's alpha was 0.81 .

Medical Outcomes Short Form 36-Health Survey (SF36). The physical aspect of quality of life was measured 
by the physical component summary (PCS) of the Taiwanese version of the SF-36. Eight domains comprise physical function, role limitation caused by physical problems, role limitation caused by emotional problems, social function, bodily pain, mental health, vitality, and general health. These eight domains can fall into either the PCS or the mental component summary (MCS). The score of each domain ranges from 0 to 100, with higher scores indicating a higher quality of life. For the purpose of this study, only four domains of the PCS were considered: physical function, role limitation caused by physical problems, bodily pain, and general health [33]. A score of $>60$ was identified as indicating a good quality of life, using norming standardized scores based on Taiwan-specific scoring algorithms [34]. Validity and reliability of the Taiwan version of the SF-36 are considered good, and the SF-36 has been used with cardiac patients [25]. In the present study, Cronbach’s alpha was 0.863.

\subsection{Data Collection}

The researcher invited potential patients from the cardiac ward. When a patient agreed to participate, a consent form was given to him or her, and the researcher, in person, assured the participant of the confidentiality of his or her information as well as the right of the participant to withdraw from the study at any time without negative consequences. The researcher assisted each participant in completing the demographic questionnaire, SACSI, and STAI prior to discharge. Participants were asked to recall the anxiety level during heart attack when they filled in STAI. One month later, all participants received the SF-36, along with a stamped, addressed envelope by which to mail the form back after completion. A total of 105 participants were recruited, of whom 100 completed the study.

\subsection{Data Analysis}

CART was run with S-Plus 6.2 software (Insightful Inc., New York, NY, USA). The Gini method was used to establish an optimal tree. The first step of CART is to build the tree by splitting the parent node into child nodes. The second step is to stop the process of growing the tree. The minimum contact points sets at 10 and 5 and the deviance of the minimum node sets at 0.1 as stop condition. The third step is to prune the tree to create a simple tree with important nodes. The final step is to select the optimal tree that fits the information in the database [26,27,35]. In addition, SPSS, Version 17.0, software (SPSS Inc, Chicago, IL, USA) was used to run stepwise regression. These two statistical approaches were used to determine predictors of the dependent variable. The $p$ value was set at $<0.05$.

\section{RESULTS}

\subsection{Demographics}

As seen in Table 1, the mean age was 62.26, and males accounted for $75 \%$ of participants. Most of participants (96\%) took care of themselves, 60 (60\%) patients were non-smokers, and 77 (77\%) patients did not drink alcohol. More than half (61\%) of the patients never or seldom

Table 1. Demographics ( $=100)$.

\begin{tabular}{|c|c|c|c|}
\hline Variables & $\mathrm{n}$ & $\%$ & Mean \pm SD \\
\hline Age & & & $62.26 \pm 10.725$ \\
\hline \multicolumn{4}{|l|}{ Gender } \\
\hline Male & 75 & 75.0 & \\
\hline Female & 25 & 25.0 & \\
\hline \multicolumn{4}{|l|}{ Education } \\
\hline Below elementary & 35 & 35.0 & \\
\hline Junior/High school & 31 & 31.0 & \\
\hline AA/Bachelor & 27 & 27.0 & \\
\hline Graduate school or above & 7 & 7.0 & \\
\hline \multicolumn{4}{|l|}{ Religious } \\
\hline None & 35 & 35.0 & \\
\hline Buddhism/Dao & 61 & 61.0 & \\
\hline Christian/Catholic & 4 & 4.0 & \\
\hline \multicolumn{4}{|l|}{ Employee status } \\
\hline None & 61 & 61.0 & \\
\hline Yes & 39 & 39 & \\
\hline \multicolumn{4}{|l|}{ Marital status } \\
\hline Married & 89 & 89.0 & \\
\hline Other & 11 & 11.0 & \\
\hline \multicolumn{4}{|l|}{ Living status } \\
\hline With family & 94 & 94.0 & \\
\hline Alone or other & 6 & 6.0 & \\
\hline \multicolumn{4}{|l|}{ Primary care provider } \\
\hline Self & 66 & $66.0 \%$ & \\
\hline Family/other & 34 & $34.0 \%$ & \\
\hline \multicolumn{4}{|l|}{ Tobacco use } \\
\hline Non-smoker & 60 & 60.0 & \\
\hline Quit & 20 & 20.0 & \\
\hline Habitual & 20 & 20.0 & \\
\hline \multicolumn{4}{|l|}{ Alcohol use } \\
\hline Non & 77 & 77.0 & \\
\hline Quit & 7 & 7.0 & \\
\hline Habitual & 16 & 16.0 & \\
\hline \multicolumn{4}{|l|}{ Exercise habit } \\
\hline None & 49 & 49.0 & \\
\hline Non-regular & 12 & 12.0 & \\
\hline Regular & 39 & 39.0 & \\
\hline Family support & & & $9.26 \pm 1.307$ \\
\hline Environment support & & & $8.91 \pm 1.741$ \\
\hline CCI & & & $3.44 \pm 0.912$ \\
\hline
\end{tabular}


exercise. The mean for the CCI was 2.58. The majority of the participants perceived their family support and environment support as good.

ACS symptoms, anxiety status, and physical component of quality of life patients of both genders experienced certain ACS symptoms, including chest pain/discomfort (95\%), difficulty breathing (47\%), and sweating (34\%). Concerning severity, $71 \%$ of participants expressed severe or very severe chest pain/discomfort and $39 \%$ expressed severe or very severe difficulty in breathing. The most frequent locations for pain were center of the chest (74\%), left side of the chest (54\%), left shoulder (20\%), and left arm (17\%). In addition, heaviness (57\%), tightness (23\%), and pressure (12\%) were frequently used terms to describe the pain/discomfort. In regard to gender, more women had chest pain/discomfort, difficult breathing, shortness of breath, and sweating, as compared to men. Women also experienced higher level of severe vomiting and shortness of breath, as compared to men.

In regard to anxiety, the mean score for the state of anxiety was 49.36 and, for trait anxiety, 43.83. Notably, $85 \%$ of participants experienced moderate or a severe state of anxiety, and $68 \%$ had moderate or severe trait anxiety when their symptoms occurred. Women had higher scores for trait anxiety, but the difference was not statistically significant. For the physical aspects of the quality of life, the mean score was 49.75 , indicating that participants did not have a good quality of life.

\subsection{CART Analysis}

PCS was identified by CART analysis as the dependent variable. Figure 1 presents a classification tree that shows that trait anxiety and exercise habits were selected as predictive variables. The first child node arose from a cut point of 51.5 for trait anxiety, and exercise habits split the node into two child nodes; therefore, four terminal nodes were established.

There were 78 patients whose trait anxiety score was lower than 51.5, and, among them, 45 patients had a regular exercise habit, yielding a mean score of 51.21 for PCS, indicating a good physical aspect of quality of life. The other 33 patients did not exercise regularly and obtained a mean score of 45.82 for PCS. In addition, there were 22 patients whose trait anxiety score was over 51.5, and, among them, 6 patients had a regular exercise habit, yielding a mean score of 44.82 for PCS. The other 16 patients did not exercise regularly and, thus, had a mean score of 33.76 for PCS.

\subsection{Regression Analysis}

Stepwise regression was used to determine the predictors of PCS. As seen in Table 2, trait anxiety and exercise habits were the most significant predictors, explaining $33.8 \%$ of the variance in PCS. In addition, sweating, new onset cough, self-perceived family support, and state anxiety also predicted better quality of life.

\section{DISCUSSION}

The results of the present study showed that chest pain/ discomfort is the main symptom of angina, which is consistent with previous research [4]. Additionally, more women had chest pain/discomfort, difficulty breathing, shortness of breath, and sweating, as compared to men. Women experienced higher-level severity of vomiting and shortness of breath, as compared to men. The differences in symptoms between genders were somewhat different from what is seen in Western studies, which shows

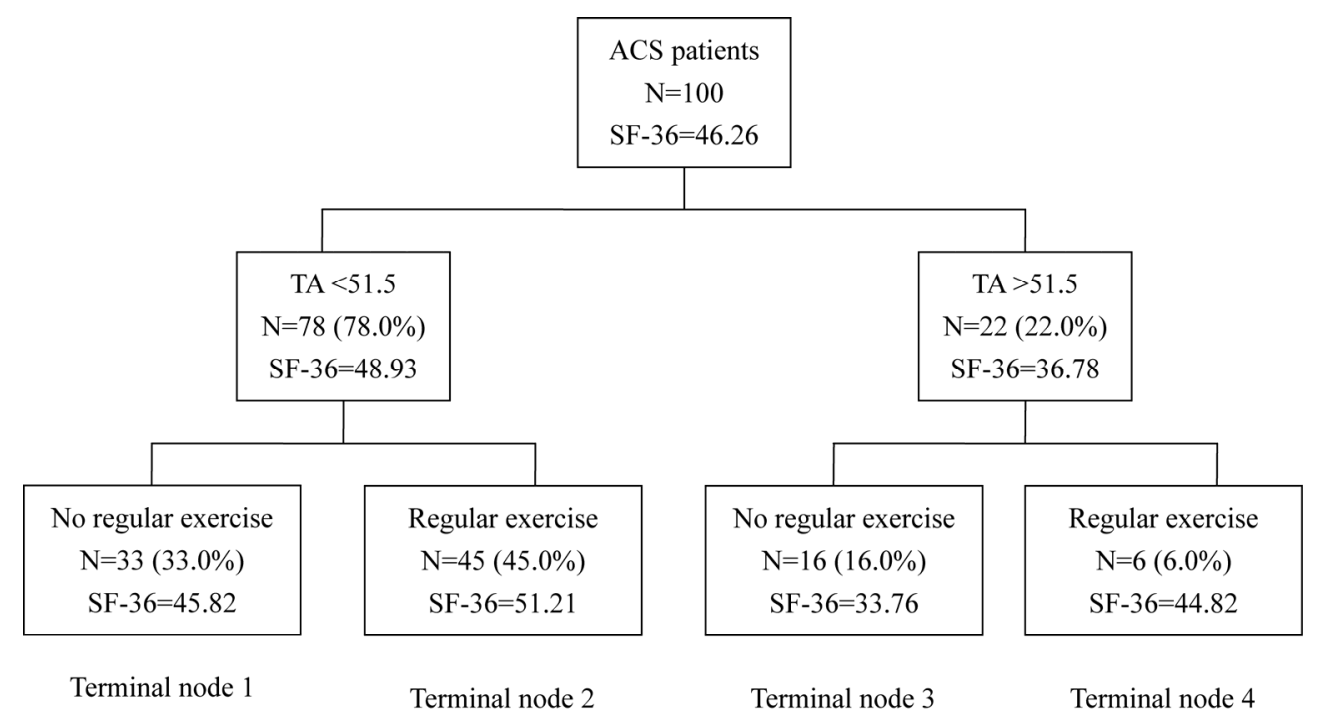

Figure 1. Decision tree of physical components' summary for the quality of life TA = Trait Anxiety. 
Table 2. Predictors of PCS (Stepwise regression).

\begin{tabular}{rlccccc}
\hline \multirow{2}{*}{ Variable } & \multicolumn{5}{c}{ Physical summary component } \\
\cline { 2 - 6 } & & $\beta$ & $R^{2}$ & Adj $R^{2}$ & $F$ & $0.000^{* * *}$ \\
\hline 1. Trait anxiety & -0.455 & 0.207 & 0.199 & 250.543 & $0.000^{* * *}$ \\
2. & Exercise habit & 0.366 & 0.338 & 0.325 & 190.304 & $0.034^{*}$ \\
3. State anxiety & -0.383 & 0.369 & 0.349 & 40.605 & $0.026^{*}$ \\
4. Sweating & -0.183 & 0.400 & 0.381 & 50.148 & $0.030^{*}$ \\
5. Family support & 0.179 & 0.429 & 0.405 & 40.835 & $0.036^{*}$ \\
6. & New onset cough & -0.163 & 0.455 & 0.426 & 40.531 & \\
\hline
\end{tabular}

${ }^{*} p<0.05,{ }^{* *} p<0.01,{ }^{* * *} p<0.001$.

that women experience more back pain, indigestion, palpitation, vomiting, fatigue, and numbness $[4,5]$. Both the present study and western studies show that women had more associated symptoms than did men. An understanding of gender difference in symptoms can provide clinicians with additional means to identify a cardiac event. The potential reasons for the differences between the findings of the present study and those of Western studies warrant further investigation.

The present study showed that, when symptoms occurred, $85 \%$ of participants experienced a state of anxiety and $68 \%$ had trait anxiety. The level of anxiety in our study was higher than that of the Koivula et al. (2001) [10], a study of patients waiting for cardiac surgery. In the present study, women experienced higher anxiety, a finding that is in keeping with previous research [15]. The anxiety may come from uncertainty and the "unknowns" associated with the disease [7]. Thus, increasing patients' knowledge of their disease and educating them to self-manage or to seek help is very important.

Certain pathophysiology mechanisms were involved in anxiety responds which might affect one's physical aspect of quality of life. Anxiety was associated with excessive of sympathetic nervous system (SNS) activation, which may result in poor cardiac outcomes. SNS activation contributes to platelet aggregation, volume contraction, recurrent thrombus formation, electric instability and endothelial dysfunction were reported [36]. Previous research also revealed compare to non-anxious patients, patients suffering from anxiety are generally more sensitive to physiologic changes which might have negative impact on recovery [37].

CART analysis uses binary recursive partitioning to split the original node into two nodes. Rood node was first split by trait anxiety, which indicating trait anxiety is the most significant independent variable and the second child node was split down by exercise habit. This process repeats until an optimal tree, for which each terminal node indicates a specific pattern of subgroups, is established. All of the information in the database was able to be analyzed in a decision tree model, rather than through linear regression, which usually selects certain indepen- dent variables based on the literature or clinical experience [26,27].

According to CART analysis, among ACS patients, less trait anxiety and a regular exercise habit predicted a better quality of life. The symptoms of ACS, however, were not selected, which indicated that trait anxiety and a regular exercise habit had a greater contribution to the quality of life than did the symptoms that ACS patient experienced. CART analysis is simple and clear, which are considered its greatest strength. Nevertheless, the results based on CART might be difficult to compare with the results of other studies because previous research that uses CART analysis to determine predictors of ACS is very limited. In such studies, linear regression is more commonly used.

In addition to trait anxiety and a regular exercise habit, sweating, new onset cough, self-perceived family support, and a state of anxiety were identified as significant predictors of better quality of life in linear regression model. These predictors were identified and their contribution to the dependent variable was determined through the value of the coefficient. Anxiety, exercise, and family support have been reported as important contributors to better quality of life. As such, the results of the present study support those of previous studies [19,23-25,38,39]. Our study's result on the impact of sweating and new onset of coughing on the quality of life, is a new finding. It may be that patients' experiencing a cough for the first time results in anxiety over the presence of a new symptom. The sweating, then, is a response to this anxiety. Such discomfort and anxiety could decrease quality of life.

In short, each approach presented had its advantages and disadvantages. CART analysis is appropriate for medical/clinical use as a means to determine the characteristics of good outcomes. However, the decision tree was developed to only four terminal nodes, and some factors, such demographics and symptoms of ACS were not selected to be part of the tree. Unlike CART, linear regression is a parametric analysis method and requires a normal distribution of the database. The benefits of linear regression are that it is widely used, accepted, and un- 
derstood. In short, using both CART and linear regression for clinical data is a good way to analyze the results from different statistical approaches, yielding valuable information for clinical use.

\section{LIMITATIONS AND RECOMMENDATIONS}

The contribution of anxiety and ACS symptoms during a cardiac event to the quality of life one month later was examined in this study. Nevertheless, what occurred during one month later was not followed or recorded. Thus, related medical treatment could have influenced the outcomes. Therefore, conducting face-to-face interviews a month after the cardiac event to discover how the patients felt is recommended. In addition, NYHA (New York Heart Association) function status should be assessed and collected since large or recurrent infraction may lead to heart failure. Anxiety was measured only at an early stage, rather than a long-term follow up after discharge. Patients might have the same, decreased, or increased levels of anxiety, which could potentially affect their quality of life. Thus, the collection of data at different points of time after discharge is recommended to identify healthcare needs at specific times. Another limitation is the low reliability of the SACSI, which might have been due to the unfamiliar terms used to describe different characteristics of pain. Employing pilot testing and an expert panel discussion as a means to review or delete terms on the SACSI is recommended. In addition, samples need to contain a greater number of women and more participants from a variety of locations to allow for analysis of gender and for the effect of menopause on these variables.

\section{CONCLUSION}

Two important new findings were reported. First, anxiety is a most significant predictor as well as a stronger predictor than the symptoms of ACS for quality of life. Anxiety level that patient experienced at the time heart attack occurred can predict quality of life a month later. Second, the majority of ACS patients experienced a moderate to high level of anxiety during a heart attack. To reduce anxiety and increase the quality of life, education should focus on what could happen in a cardiac event and what to do when it occurs in ACS patients or those who are at risk of ACS. While it is important for healthcare professionals to understand these aspects of ACS, it is just as important for them to educate their patients. This would be a good first step toward patients' having the knowledge needed to reduce their anxiety.

\section{REFERENCES}

[1] Lloyd-Jones, D., Adams, R., Carnethon, M., et al. (2009)
Heart Disease and Strok Statistic_-2009 Update: A report from the American Heart Association statistics committee and stroke statistic. Circulation, 119, 21-181. doi:10.1161/CIRCULATIONAHA.108.191261

[2] Department of Health, Executive Yuan. R.O.C (Taiwan) (2010) Average number of cases occurred every day. http://www.doh.gov.tw/CHT2006/DM/DM2_2.aspx?now _fod_list_no $=11964 \&$ class_no $=440 \&$ level_no $=5$.

[3] Kumar, A. and Cannon, C.P. (2009) Acute coronary syndromes: Diagnosis and management, part I. Mayo Clinic Proceedings, 84, 917-938. doi:10.4065/84.10.917

[4] DeVon, H.A., Ryan, C.J., Ochs, A.L., et al. (2008) Symptoms across the continuum of acute coronary syndromes: Differences between women and men. American Journal of Critical Care, 17, 14-24.

[5] Shin, J.Y., Martin, R. and Suls, J. (2009) Meta-analytic evaluation of gender differences and symptom measurement strategies in acute coronary syndromes. Heart \& Lung, 39, 283-295. doi:10.1016/j.hrtlng.2009.10.010

[6] Koivula, M., Hautamaki-Lamminen, K. and Astedt-Kurki, P. (2009) Predictors of fear and anxiety nine years after coronary artery bypass grafting. Journal of Advanced Nursing, 66, 595-606. doi:10.1111/j.1365-2648.2009.05230.x

[7] Gudykunst, W.B. and Nishida, T. (2001) Anxiety, uncertainty, and perceived effectiveness of communication across relationships and cultures. International Journal of Intercultural Relations, 25, 55-71. doi:10.1016/S0147-1767(00)00042-0

[8] Wang, W., Chair, S.Y., Thompson, D.R., et al. (2009) A psychometric evaluation of the Chinese version of the Hospital Anxiety and Depression Scale in patients with coronary heart disease. Journal of Clinical Nursing, 18, 1908-1915. doi:10.1111/j.1365-2702.2008.02736.x

[9] Townsend, M.C. (2008) Essentials of psychiatric mental health nursing: Concepts of care in evidence-based practice. Davis Company, Philadelphia.

[10] Koivula, M., Paunonen-Ilmonen, M., Tarkka, M.T., et al. (2001) Fear and anxiety in patients awaiting coronary artery bypass grafting. Heart \& Lung, 30, 302-311. doi:10.1067/mhl.2001.116134

[11] Karlsson, I., Berglin, E. and Larsson, P.A. (2000) Sense of coherence: Quality of life before and after coronary artery bypass surgery-A longitudinal study. Journal of Advanced Nursing, 30, 1383-1392. doi:10.1046/j.1365-2648.2000.01408.x

[12] Koivula, M., Tarkka, M.T., Tarkka, M., et al. (2002) Fear and anxiety in patients at different time-points in the coronary artery bypass process. International Journal of Nursing Studies, 39, 811-822. doi:10.1016/S0020-7489(02)00022-6

[13] Andrew, M.J., Baker, R.A., Kneebone, A.C., et al. (2000) Mood state as a predictor of neuropsychological deficits following cardiac surgery. Journal of Psychosomatic Research, 48, 537-546. doi:10.1016/S0022-3999(00)00089-1

[14] Hoyera, J., Eifertb, G.H., Einslec, F., et al. (2008) Heartfocused anxiety before and after cardiac surgery. Journal 
of Psychosomatic Research, 64, 291-297. doi:10.1016/j.jpsychores.2007.09.009

[15] Tung, H.H., Hunter, A., Wei, J., et al. (2009) Gender differences in coping and anxiety in patients after coronary artery bypass graft surgery in Taiwan. Heart \& Lung, 38, 469-479. doi:10.1016/j.hrtlng.2009.01.006

[16] Chan, D.S.K., Chau, J.P.C. and Chang, A.M. (2005) Acute coronary syndromes cardiac rehabilitation programmers and quality of life. Journal of Advanced Nursing, 49, 591-599. doi:10.1111/j.1365-2648.2004.03334.X

[17] Treat-Jacobson, D.J. and Lindquist, R. (2007) Exercise, quality of life, and symptoms in men and women five to six years after coronary artery bypass graft surgery. Heart \& Lung, 36, 387-297. doi:10.1016/j.hrtlng.2007.01.002

[18] Rantanen, A., Kaunonen, M., Sintonen, H., et al. (2008) Factors associated with health-related quality of life in patients and significant others one month after coronary artery bypass grafting. Journal of Clinical Nursing, 17, 1742-1753. doi:10.1111/j.1365-2702.2007.02195.x

[19] Ulvik, B., Nygard, O., Hanestad, B.R., et al. (2008) Associated between disease severity, coping and dimensions of health-related quality of life in patients admitted for elective coronary angiography-A cross sectional study. Health and Quality of Life Outcomes, 6, 38. doi:10.1186/1477-7525-6-38

[20] Mayou, R.A., Gill, D., Thompson, D.R., et al. (2000) Depression and anxiety as predictors of outcome after myocardial infarction. Psychosomatic Medicine, 62, 212219.

[21] Szekely, A., Balog, P., Benko, E., et al. (2007) Anxiety predicts mortality and morbidity after coronary artery and valve surgery-A 4-year follow-up study. Psychosomatic Medicine, 69, 625-631. doi:10.1097/PSY.0b013e31814b8c0f

[22] Benedetto, M.D., Lindner, H., Hare, D.L., et al. (2007) The role of coping, anxiety, and stress in depression postacute coronary syndrome. Psychology, Health \& Medicine, 12, 460-469. doi:10.1080/13548500601109334

[23] Tully, P.J., Baker, R.A., Turnbull, D.A., et al. (2009) Negative emotion and quality of life six mouths after cardiac surgery: The dominant role of depression not anxiety symptoms. Journal of Behavioral Medicine, 32, 512-522. doi:10.1007/s10865-009-9225-4

[24] Carmeli, E., Barak, S., Morad, M., et al. (2009) Physical exercise can reduce anxiety and improve quality of life among adults with intellectual disability. International SportMed Journal, 10, 77-85.

[25] Tung, H.H., Hunter, A. and Wei, J. (2008) Coping, anxiety and quality of life after coronary artery bypass graft surgery. Journal of Advanced Nursing, 61, 651-663. doi:10.1111/j.1365-2648.2007.04557.x

[26] Lewis, R.J. (2000) An introduction to classification and regression tree (CART) analysis. Annual Meeting of the Society for Academic Emergency Medicine, San Francisco.

[27] Phelps, M.C. and Merkle, E.C. (2008) Classification and regression tree as alternative to regression. Proceedings of the $4^{\text {th }}$ GRASP Symposium, Wichita State University.

[28] Sheu, S. (2001) Uncertainty and anxiety in patients with initial attack of myocardial infarction: The effect of coping methods. Nursing Research, 9, 159-171.

[29] Souza, R.C., Pinheiro, R.S., Coeli, C.M., et al. (2006) The Charlson comorbidity index (CCI) for adjustment of hip fracture mortality in the elderly: Analysis of the importance of recording econdary diagnoses. Cadernos de Saúde Pública, 24, 315-322.

[30] McGregor, J.C., Kim, P.W., Perencevich, E.N., et al. (2005) Utility of the chronic disease score and charlson comorbidity index as comorbidity ceasures for use in epidemiologic studies of antibiotic-resistant organisms. American Journal of Epidemiology, 161, 483-493. doi:10.1093/aje/kwi068

[31] Seo, H.J., Yoon, S.J., Lee, S.I., et al. (2010) A comparison of the Charlson comorbidity index derived from medical records and claims data from patients undergoing lung cancer surgery in Korea: A population-based investigation. BMC Health Services Research, 10, 236. doi:10.1186/1472-6963-10-236

[32] McGregor, J.C., Kim, P.W., Perencevich, E.N., Bradham, D.D., Furuno, J.P., Kaye, K.S., et al. (2005) Utility of the chronic disease score and Charlson comorbidity index as comorbidity measures for use in epidemiologic studies of antibiotic-resistant organisms. American Journal of Epidemiology, 161, 483-493. doi:10.1093/aje/kwi068

[33] Ware, J.E.J., Kosinski, M. and Keller, S.D. (1994) SF-36 Physical \& mental health summary scales: A user's manual. Health Assessment Lab. New England Medical Center, Boston.

[34] Tseng, H.M., Lu, J.F., Tsai, Y.J. (2003) Assessment of health-related quality of life in Taiwan (II): Norming and validation of SF-36 Taiwan version. Taiwan Journal of Public Health, 22, 512-518.

[35] Brian, S.E. (2004) Morden medical statistics. Hodder Arnold, Oxford, New York.

[36] Konstam, V., Moser, D.K. and De Jong, M.J. (2005). Depression and anxiety in heart failure. Journal of Heart Failure, 11, 455-463.

[37] Hoehn-Sark, R., McLeod, D., Funderburk, F., et al. (2004). Somatic symptoms and physiologic responses in generalized anxiety disorder and panic disorder. Archives of General Psychiatry, 61, 913-921. doi:10.1001/archpsyc.61.9.913

[38] Tung, H.H., Chen, Y.C., Wei, J., et al. (2010) Leisure physical activity and quality of life after coronary artery bypass graft surgery for patients with metabolic syndrome in Taiwan. Heart \& Lung, 39, 410-420. doi:10.1016/j.hrtlng.2009.10.004

[39] Tung, H.H., Chen, H.L., Wei, J., et al. (2010) Predictors of quality of life in heart-transplant recipients in Taiwan. Heart \& Lung, 40, 320-330. doi:10.1016/j.hrtlng.2009.11.003 